TAIWANESE JOURNAL OF MATHEMATICS

Vol. 15, No. 2, pp. 633-658, April 2011

This paper is available online at http://www.tjm.nsysu.edu.tw/

\title{
COMPACTNESS FOR COMMUTATORS OF MARCINKIEWICZ INTEGRALS IN MORREY SPACES
}

\author{
Yanping Chen, Yong Ding* and Xinxia Wang
}

\begin{abstract}
In this paper the authors give a characterization of the compactness for the commutator $\left[b, \mu_{\Omega}\right]$ in the Morrey spaces $L^{p, \lambda}\left(\mathbb{R}^{n}\right)$, where $\mu_{\Omega}$ denotes the Marcinkiewicz integral. More precisely, the authors prove that if $b \in$ $\operatorname{VMO}\left(\mathbb{R}^{n}\right)$, the $\operatorname{BMO}\left(\mathbb{R}^{n}\right)$-closure of $C_{c}^{\infty}\left(\mathbb{R}^{n}\right)$, then the commutators $\left[b, \mu_{\Omega}\right]$ is a compact operator in the Morrey spaces $L^{p, \lambda}\left(\mathbb{R}^{n}\right)$ for $1<p<\infty$ and $0<\lambda<n$. Conversely, if $b \in \operatorname{BMO}\left(\mathbb{R}^{n}\right)$ and $\left[b, \mu_{\Omega}\right]$ is a compact operator in $L^{p, \lambda}\left(\mathbb{R}^{n}\right)$ for some $p \in(1, \infty)$ and $\lambda \in(0, n)$, then $b \in \operatorname{VMO}\left(\mathbb{R}^{n}\right)$. In the above results, the kernel function $\Omega$ of the operator $\mu_{\Omega}$ is assumed to satisfy a very weak condition on $S^{n-1}$.
\end{abstract}

\section{INTRODUCTION}

Let $S^{n-1}$ be the unit sphere in $\mathbb{R}^{n}$ equipped with the Lebesgue measure $d \sigma$. In 1958, Stein [14] defined the Marcinkiewicz integral of high dimension. Suppose that $\Omega$ satisfies the following conditions:

(a) $\Omega$ is homogeneous function of degree zero on $\mathbb{R}^{n} \backslash\{0\}$, i.e.

$$
\Omega(t x)=\Omega(x) \text { for any } t>0 \text { and } x \in \mathbb{R}^{n} \backslash\{0\} .
$$

(b) $\Omega$ has mean zero on $S^{n-1}$, i.e.

$$
\int_{S^{n-1}} \Omega\left(x^{\prime}\right) d \sigma\left(x^{\prime}\right)=0 .
$$

(c) $\Omega \in \operatorname{Lip}\left(S^{n-1}\right)$, i.e.

Received April 9, 2008, accepted September 22, 2009.

Communicated by Yongsheng Han.

2000 Mathematics Subject Classification: 42B30, 42B99.

Key words and phrases: Marcinkiewicz integrals, Commutators, Compactess, VMO, Morrey space. The research was supported by NSF of China (Grants: 10931001 and 10901017) and SRFDP of China (Grant: 20090003110018).

*Corresponding author. 


$$
\left|\Omega\left(x^{\prime}\right)-\Omega\left(y^{\prime}\right)\right| \leq\left|x^{\prime}-y^{\prime}\right| \quad \text { for any } \quad x^{\prime}, y^{\prime} \in S^{n-1} .
$$

Then the Marcinkiewicz integral $\mu_{\Omega}$ is defined by

$$
\mu_{\Omega}(f)(x)=\left(\int_{0}^{\infty}\left|F_{\Omega, t}(x)\right|^{2} \frac{d t}{t^{3}}\right)^{\frac{1}{2}}
$$

where

$$
F_{\Omega, t}(x)=\int_{|x-y| \leq t} \frac{\Omega(x-y)}{|x-y|^{n-1}} f(y) d y .
$$

The Marcinkiewicz integral $\mu_{\Omega}$ is essentially a Littlewood-Paley $g$-function. In fact, if taking

$$
\varphi(x)=\Omega(x)|x|^{-n+1} \chi_{\{|x| \leq 1\}}(x)
$$

and $\varphi_{t}(x)=t^{-n} \varphi\left(\frac{x}{t}\right)$ for $t>0$, then

$$
\mu_{\Omega} f(x)=\left(\int_{0}^{\infty}\left|\varphi_{t} * f(x)\right|^{2} \frac{d t}{t}\right)^{1 / 2} .
$$

In 1958, Stein [14] gave the weak $(1,1)$ boundedness and $L^{p}$ boundedness of $\mu_{\Omega}$ for $1<p \leq 2$. In 1961, Benedeck, Calderon and Panzone [1] proved that if replacing the Lipschitz condition (1.3) by $\Omega \in C^{1}\left(S^{n-1}\right)$, then $\mu_{\Omega}$ is bounded operator in $L^{p}$ for $1<p<\infty$. In 2000, Ding, Fan and Pan [8] showed further that the smoothness assumed on $\Omega$ is not necessary for the $L^{p}(1<p<\infty)$ boundedness of $\mu_{\Omega}$.

Theorem A. ([8]). If $\Omega \in H^{1}\left(S^{n-1}\right)$ satisfies (1.1) and (1.2), then $\mu_{\Omega}$ is of type $(p, p)$ for $1<p<\infty$, where $H^{1}\left(S^{n-1}\right)$ denotes the Hardy space on $S^{n-1}$. See [6] for the definition and properties of $H^{1}\left(S^{n-1}\right)$.

Remark 1.1. There are the following including relationship on $S^{n-1}$ :

$$
C^{1}\left(S^{n-1}\right) \subset \operatorname{Lip}\left(S^{n-1}\right) \subset L^{q}\left(S^{n-1}\right)(1<q \leq \infty) \subset L \log ^{+} L\left(S^{n-1}\right) \subset H^{1}\left(S^{n-1}\right),
$$

and all inclusions above are proper. On the other hand, In 1976, in their famous paper [5], Coifman, Rochberg and Weiss gave an $L^{p}$-boundedness characterization of the commutator $[b, T]$ of the Calderon-Zygmund singular integral operator $T$.

Theorem B. ([5]). Suppose that $\Omega$ satisfies (1.1), (1.2) and (1.3).

(i) If $b \in \operatorname{BMO}\left(\mathbb{R}^{n}\right)$, then $[b, T]$ is bounded in $L^{p}\left(\mathbb{R}^{n}\right)$ for all $1<p<\infty$.

(ii) If $\left\{\left[b, R_{j}\right]\right\}_{j=1}^{n}$ are bounded in $L^{p}\left(\mathbb{R}^{n}\right)$ for some $p, 1<p<\infty$, then $b \in$ $\mathrm{BMO}\left(\mathbb{R}^{n}\right)$, where $R_{j}(j=1, \cdots, n$, ) denotes the jth Reisz transform. 
In 1978, Uchiyama [18] and Janson [11] extended Theorem B independently. The results in [18] and [11] show that the Reisz transform $R_{j}$ in the conclusion (ii) of Theorem B may be replaced by the Calderon-Zygmund singular integral operator $T$.

In the same paper [18], Uchiyama considered also the characterization of the commutator $[b, T]$ is a compact operator in the Lebesgue space $L^{p}\left(\mathbb{R}^{n}\right)$. Denote by $\operatorname{VMO}\left(\mathbb{R}^{n}\right)$ the BMO-closure of $C_{c}^{\infty}\left(\mathbb{R}^{n}\right)$, where $C_{c}^{\infty}\left(\mathbb{R}^{n}\right)$ is the set of $C^{\infty}\left(\mathbb{R}^{n}\right)$ functions with compact support. Uchiyama proved the following conclusions:

Theorem C. ([18]). Suppose that $\Omega$ satisfies (1.1), (1.2) and (1.3).

(i) If $b \in \mathrm{VMO}\left(\mathbb{R}^{n}\right)$, then $[b, T]$ is compact in $L^{p}\left(\mathbb{R}^{n}\right)$ for all $1<p<\infty$.

(ii) If $[b, T]$ is a compact operator in $L^{p}\left(\mathbb{R}^{n}\right)$ for some $p, 1<p<\infty$, then $b \in \operatorname{VMO}\left(\mathbb{R}^{n}\right)$.

Naturally, one may ask the question whether hold still the conclusions in Theorems $\mathrm{B}$ and $\mathrm{C}$ if replacing $[b, T]$ by the commutator $\left[b, \mu_{\Omega}\right]$ of the Marcinkiewicz integral and the space $L^{p}\left(\mathbb{R}^{n}\right)$ by the Morrey space $L^{p, \lambda}\left(\mathbb{R}^{n}\right)$, respectively. In this paper and the forthcoming paper, we will give a positive answer to this question. In fact, we will get similar conclusions to those of Theorems $\mathrm{B}$ and $\mathrm{C}$ under more weaker conditions than those in Theorems B and C.

Before stating some results, let us recall some definitions. For $b \in L_{\mathrm{loc}}\left(\mathbb{R}^{n}\right)$, the commutator $\left[b, \mu_{\Omega}\right]$ formed by $b$ and the Marcinkiewicz integral $\mu_{\Omega}$ is defined by

$$
\left[b, \mu_{\Omega}\right] f(x)=\left(\int_{0}^{\infty}\left|\int_{|x-y| \leq t} \frac{\Omega(x-y)}{|x-y|^{n-1}}(b(x)-b(y)) f(y) d y\right|^{2} \frac{d t}{t^{3}}\right)^{1 / 2} .
$$

For $\Omega \in L^{q}\left(S^{n-1}\right), q \geq 1$, the integral modulus $\omega_{q}(\delta)$ of continuity of order $q$ of $\Omega$ is defined by

$$
\omega_{q}(\delta)=\sup _{\|\tau\| \leq \delta}\left(\int_{S^{n-1}}\left|\Omega\left(\tau x^{\prime}\right)-\Omega\left(x^{\prime}\right)\right|^{q} d \sigma\left(x^{\prime}\right)\right)^{1 / q},
$$

where $\tau$ denotes the rotation in $\mathbb{R}^{n}$ and $\|\tau\|=\sup _{x^{\prime} \in S^{n-1}}\left|\tau x^{\prime}-x^{\prime}\right|$. The function $\Omega$ is said to satisfy the $L^{q}$-Dini condition if

$$
\int_{0}^{1} \frac{\omega_{q}(\delta)}{\delta} d \delta<\infty
$$

Recently, the first and second authors of this paper gave a characterization of the compactness of the commutators for $\mu_{\Omega}$ in $L^{p}\left(\mathbb{R}^{n}\right)$ for $1<p<\infty$.

Theorem D. ([3]). Suppose that $\Omega$ satisfies (1.1) and (1.2). 
(i) If there exist two constants $C_{1}>0$ and $\gamma>1$ such that

$$
\left|\Omega\left(x^{\prime}\right)-\Omega\left(y^{\prime}\right)\right| \leq \frac{C_{1}}{\left(\log \frac{2}{\left|x^{\prime}-y^{\prime}\right|}\right)^{\gamma}} \quad \text { for any } \quad x^{\prime}, y^{\prime} \in S^{n-1},
$$

and the commutator $\left[b, \mu_{\Omega}\right]$ is a compact operator in $L^{p}\left(\mathbb{R}^{n}\right)$ for some $p(1<$ $p<\infty)$, then $b \in \operatorname{VMO}\left(\mathbb{R}^{n}\right)$.

(ii) If $\Omega \in L^{q}\left(S^{n-1}\right)(q>1)$ satisfying the following condition:

$$
\int_{0}^{1} \frac{\omega_{q}(\delta)}{\delta}(1+|\log \delta|) d \delta<\infty,
$$

then for $b \in \operatorname{VMO}\left(\mathbb{R}^{n}\right)$, the commutator $\left[b, \mu_{\Omega}\right]$ is a compact operator in $L^{p}\left(\mathbb{R}^{n}\right)$ for $1<p<\infty$.

The purpose of this paper is to give a characterization of the compactness of the commutators $\left[b, \mu_{\Omega}\right]$ in the Morrey space $L^{p, \lambda}\left(\mathbb{R}^{n}\right)$. For $1 \leq p<\infty$ and $0<\lambda<n$, the Morrey space $L^{p, \lambda}\left(\mathbb{R}^{n}\right)$ is defined by

$$
L^{p, \lambda}\left(\mathbb{R}^{n}\right)=\left\{f \in L_{l o c}^{p}:\|f\|_{p, \lambda}<\infty\right\},
$$

where

$$
\|f\|_{p, \lambda}^{p}=\sup _{\substack{y \in \mathbb{R}^{n} \\ r>0}} \frac{1}{r^{\lambda}} \int_{B(y, r)}|f(x)|^{p} d x
$$

and $B(y, r)$ denotes the ball centered at $y$ and with radius $r>0$. The spaces $L^{p, \lambda}\left(\mathbb{R}^{n}\right)$ becomes a Banach space with norm $\|\cdot\|_{p, \lambda}$. Moreover, if $\lambda=0$ and $\lambda=n$, then $L^{p, 0}\left(\mathbb{R}^{n}\right)$ and $L^{p, n}\left(\mathbb{R}^{n}\right)$ coincide (with equality of norms) with the space $L^{p}\left(\mathbb{R}^{n}\right)$ and $L^{\infty}\left(\mathbb{R}^{n}\right)$, respectively.

The main results in this paper are as follows.

Theorem 1. Suppose that $\Omega$ satisfies (1.1), (1.2) and (1.4). If $0<\lambda<n$, $b \in \mathrm{BMO}\left(\mathbb{R}^{n}\right)$ and the commutator $\left[b, \mu_{\Omega}\right]$ is a compact operator from $L^{p, \lambda}\left(\mathbb{R}^{n}\right)$ to itself for some $p(1<p<\infty)$, then $b \in \operatorname{VMO}\left(\mathbb{R}^{n}\right)$.

Theorem 2. Suppose that $0<\lambda<n$ and $\Omega$ satisfies (1.1), (1.2) and (1.5) with $q>n /(n-\lambda)$. If $b \in \mathrm{VMO}\left(\mathbb{R}^{n}\right)$, then the commutator $\left[b, \mu_{\Omega}\right]$ is a compact operator in $L^{p, \lambda}\left(\mathbb{R}^{n}\right)$ for $1<p<\infty$.

It is easy to see that the condition (1.4) implies (1.5), so we may get the following corollary immediately.

Corollary 1. Suppose that $\Omega$ satisfies (1.1), (1.2) and (1.4). If $0<\lambda<n$, $1<p<\infty$ and $b \in \mathrm{BMO}\left(\mathbb{R}^{n}\right)$, then the commutator $\left[b, \mu_{\Omega}\right]$ is a compact operator in $L^{p, \lambda}\left(\mathbb{R}^{n}\right)$ if and only if $b \in \operatorname{VMO}\left(\mathbb{R}^{n}\right)$.

Throughout, the letter " $C$ " will denote (possibly different) the constants that are independent of the essential variables. 


\section{Proof of Theorem 1}

Let us begin with recalling some known conclusions.

Lemma 2.1. ([16]). If $b \in \operatorname{BMO}\left(\mathbb{R}^{n}\right), \alpha_{2}>\alpha_{1}>2, Q$ is a cube centered at $x_{0}$ and of diameter $r$, then exist positive constants $\alpha_{3}, \alpha_{4}, \alpha_{5}$ (depend on $\alpha_{1}, \alpha_{2}$ and $b)$, such that

$$
\left|\left\{\alpha_{1} r<\left|x-x_{0}\right|<\alpha_{2} r:\left|b(x)-b_{Q}\right|>v+\alpha_{3}\right\}\right| \leq \alpha_{4}|Q| e^{-\alpha_{5} v} \quad(0<v<\infty) .
$$

Lemma 2.2. ([17]). Suppose that $f(x)$ is a measurable function on $\mathbb{R}^{n}$. For $s>0$, let

$$
\lambda_{f}(s)=\left|\left\{x \in \mathbb{R}^{n}:|f(x)|>s\right\}\right|
$$

and

$$
f^{*}(t)=\inf \left\{s: \lambda_{f}(s) \leq t\right\} \text { for } t>0 .
$$

Then for any measurable set $E$ and $1 \leq p<\infty$,

$$
\int_{E}|f(x)|^{p} d x \leq \int_{0}^{|E|}\left|f^{*}(t)\right|^{p} d t
$$

Lemma 2.3. ([18]). Let $b \in \mathrm{BMO}\left(\mathbb{R}^{n}\right)$. Then $b \in \operatorname{VMO}\left(\mathbb{R}^{n}\right)$ if and only if $b$ satisfies the following three conditions:

(i) $\lim _{a \rightarrow 0} \sup _{|Q|=a} M(b, Q)=0$;

(ii) $\lim _{a \rightarrow \infty} \sup _{|Q|=a} M(b, Q)=0$;

(iii) $\lim _{|x| \rightarrow \infty} M(b, Q+x)=0$ for each $Q$.

Lemma 2.4. ([4]). Let $0<\lambda<n$. Suppose that $\Omega$ satisfies (1.1), (1.2) and $\Omega \in L^{q}\left(S^{n-1}\right)$ for $q>n /(n-\lambda), T$ is a linear or sublinear operator satisfying

$$
|T f(x)| \leq C \int_{\mathbb{R}^{n}} \frac{|\Omega(x-y)|}{|x-y|^{n}}|f(y)| d y .
$$

(i) If the operator $T$ is bounded on $L^{p}\left(\mathbb{R}^{n}\right)$ for $1<p<\infty$, then $T$ is also bounded on $L^{p, \lambda}\left(\mathbb{R}^{n}\right)$.

(ii) For $b \in \operatorname{BMO}\left(\mathbb{R}^{n}\right)$, if the commutator $[b, T]$ is bounded on $L^{p}\left(\mathbb{R}^{n}\right)$ for $1<p<\infty$, then $[b, T]$ is also bounded on $L^{p, \lambda}\left(\mathbb{R}^{n}\right)$. 
Lemma 2.5. (see [7]) If $\Omega$ satisfies conditions (1.1), (1.2) and (1.4). Let $\beta>0$. Then for $|x|>2|y|$

$$
\left|\frac{\Omega(x-y)}{|x-y|^{\beta}}-\frac{\Omega(x)}{|x|^{\beta}}\right| \leq \frac{C}{|x|^{\beta}\left(\log \frac{|x|}{|y|}\right)^{\gamma}} .
$$

Now let us return to the proof of Theorem 1. Suppose that $b \in \operatorname{BMO}\left(\mathbb{R}^{n}\right)$ and $\left[b, \mu_{\Omega}\right]$ is a compact operator in $L^{p, \lambda}\left(\mathbb{R}^{n}\right)$. By Lemma 2.3, to prove that $b \in \operatorname{VMO}\left(\mathbb{R}^{n}\right)$, it suffices to show that $b$ must satisfy the conditions (i), (ii) and (iii) in Lemma 2.3.

First, we show that if $b$ does not satisfy the condition (i) of Lemma 2.3, then $\left[b, \mu_{\Omega}\right]$ is not a compact operator in $L^{p, \lambda}\left(\mathbb{R}^{n}\right)$. By the assumption, there exist a $\zeta>0$ and a sequence of cubes $\left\{Q_{j}\left(y_{j}, q_{j}\right)\right\}_{j=1}^{\infty}$ with $\lim _{j \rightarrow \infty} q_{j}=0$ such that for every $j$

$$
M\left(b, Q_{j}\right)=\left|Q_{j}\right|^{-1} \int_{Q_{j}}\left|b(y)-b_{Q_{j}}\right| d y>\zeta .
$$

Without loss of the generality, we may assume $\|b\|_{*}=1$. Define the function sequence $\left\{f_{j}\right\}_{j=1}^{\infty}$ by

$$
f_{j}(y)=\left|Q_{j}\right|^{-(n-\lambda) /(n p)}\left[\operatorname{sgn}\left(b(y)-b_{Q_{j}}\right)-c_{0}\right] \chi_{Q_{j}}(y), \quad j=1,2, \cdots,
$$

where $c_{0}=\left|Q_{j}\right|^{-1} \int_{Q_{j}} \operatorname{sgn}\left(b(y)-b_{Q_{j}}\right) d y$. Since $\int_{Q_{j}}\left(b(y)-b_{Q_{j}}\right) d y=0$, it is easy to check that $\left|c_{0}\right|<1$ and $\left\{f_{j}\right\}$ satisfies the following properties:

$$
\begin{gathered}
\operatorname{supp} f_{j} \subset Q_{j}, \\
f_{j}(y)\left(b(y)-b_{Q_{j}}\right) \geq 0, \\
\int_{\mathbb{R}^{n}} f_{j}(y) d y=0, \\
\left|f_{j}(y)\right| \leq 2\left|Q_{j}\right|^{-(n-\lambda) /(n p)}, \quad \text { for } y \in Q_{j} .
\end{gathered}
$$

Moreover, $\left\{\left\|f_{j}\right\|_{p, \lambda}\right\}_{j=1}^{\infty}$ is bounded uniformly. In fact, denote by $B(t, r)$ any a ball in $\mathbb{R}^{n}$. If $0<r \leq q_{j}$, then

$$
\left(\frac{1}{r^{\lambda}} \int_{B(t, r)}\left|f_{j}(x)\right|^{p} d x\right)^{\frac{1}{p}} \leq C\left(\frac{r}{q_{j}}\right)^{(n-\lambda) / p} \leq C .
$$

If $r>q_{j}>0$, then 


$$
\left(\frac{1}{r^{\lambda}} \int_{B(t, r)}\left|f_{j}(x)\right|^{p} d x\right)^{\frac{1}{p}} \leq\left(\frac{1}{r^{\lambda}} \int_{Q_{j}}\left|f_{j}(x)\right|^{p} d x\right)^{\frac{1}{p}} \leq C\left(\frac{q_{j}}{r}\right)^{\frac{\lambda}{p}} \leq C .
$$

Using the Minkowski inequality, it is easy to see that

$$
\left|\mu_{\Omega}(f)(x)\right| \leq C \int_{\mathbb{R}^{n}} \frac{|\Omega(x-y)|}{|x-y|^{n}}|f(y)| d y .
$$

Moreover, $\mu_{\Omega}$ and its commutator $\left[b, \mu_{\Omega}\right]$ are both bounded on $L^{p}(1<p<\infty)$ by Theorem A and Theorem 1 in [10], respectively. Then, by Lemma 2.4, we obtain

$$
\left\|\mu_{\Omega}(f)\right\|_{p, \lambda} \leq C\|f\|_{p, \lambda}
$$

and

$$
\left\|\left[b, \mu_{\Omega}\right] f\right\|_{p, \lambda} \leq C\|b\|_{*}\|f\|_{p, \lambda} .
$$

Thus $\left\{\left[b, \mu_{\Omega}\right] f_{j}\right\}_{j=1}^{\infty}$ is also a bounded set in $L^{p, \lambda}\left(\mathbb{R}^{n}\right)$. Hence, if $\left\{\left[b, \mu_{\Omega}\right] f_{j}\right\}_{j=1}^{\infty}$ is not a pre-compact set in $L^{p, \lambda}\left(\mathbb{R}^{n}\right)$ then $\left[b, \mu_{\Omega}\right]$ is not compact operator in $L^{p, \lambda}\left(\mathbb{R}^{n}\right)$. (See [2] for the definition of the compact operator in Banach space.) To do this, it suffices to show that there exists a subsequence $\left\{\left[b, \mu_{\Omega}\right] f_{j_{k}}\right\}_{k=1}^{\infty}$, which has no any convergence subsequence in $L^{p, \lambda}\left(\mathbb{R}^{n}\right)$.

From now on, for $1 \leq i \leq 19, A_{i}$ denotes the positive constant depending only on $\Omega, p, n, \lambda, \zeta$ and $A_{k}(1 \leq k<i)$. Since $\Omega$ satisfies (1.2), then there exists an $A_{1}$ such that $0<A_{1}<1$ and

$$
\sigma\left(\left\{x^{\prime} \in S^{n-1}: \Omega\left(x^{\prime}\right) \geq \frac{2 C_{1}}{\left(\log \frac{2}{A_{1}}\right)^{\gamma}}\right\}\right)>0 .
$$

By the condition (1.4), it is easy to see that

$$
\Lambda:=\left\{x^{\prime} \in S^{n-1}: \Omega\left(x^{\prime}\right) \geq \frac{2 C_{1}}{\left(\log \frac{2}{A_{1}}\right)^{\gamma}}\right\}
$$

is a closed set. We now claim that

(2.8) if $x^{\prime} \in \Lambda$ and $y^{\prime} \in S^{n-1}$ satisfying $\left|x^{\prime}-y^{\prime}\right| \leq A_{1}, \quad$ then $\Omega\left(y^{\prime}\right) \geq \frac{C_{1}}{\left(\log \frac{2}{A_{1}}\right)^{\gamma}}$.

In fact, since

$$
\left|\Omega\left(x^{\prime}\right)-\Omega\left(y^{\prime}\right)\right| \leq \frac{C_{1}}{\left(\log \frac{2}{\left|x^{\prime}-y^{\prime}\right|}\right)^{\gamma}} \leq \frac{C_{1}}{\left(\log \frac{2}{A_{1}}\right)^{\gamma}},
$$

and note that $\Omega\left(x^{\prime}\right) \geq 2 \frac{C_{1}}{\left(\log \frac{2}{A_{1}}\right)^{\gamma}}$, we therefore get $\Omega\left(y^{\prime}\right) \geq \frac{C_{1}}{\left(\log \frac{2}{A_{1}}\right)^{\gamma}}$. Taking $A_{2}>2 / A_{1}$, if $y \in Q_{j}$ 


$$
\left|x-y_{j}\right|>A_{2}\left|y-y_{j}\right| \quad \text { for } \quad x \in\left(A_{2} Q_{j}\right)^{c} \cap\left\{x:\left(x-y_{j}\right)^{\prime} \in \Lambda\right\} .
$$

Thus by [16],

$$
\left|\left(x-y_{j}\right)^{\prime}-(x-y)^{\prime}\right| \leq \frac{2\left|y-y_{j}\right|}{\left|x-y_{j}\right|} \leq \frac{2}{A_{2}}<A_{1} .
$$

Applying (2.8), we get $\Omega\left((x-y)^{\prime}\right) \geq \frac{C_{1}}{\left(\log \frac{2}{A_{1}}\right)^{\gamma}}$. Hence, for $x \in\left(A_{2} Q_{j}\right)^{c} \cap$ $\left\{x:\left(x-y_{j}\right)^{\prime} \in \Lambda\right\}$, by $(2.1)-(2.3)$ and the Hölder inequality, and noting that $\left|x-y_{j}\right| \simeq|x-y|$, we have

$$
\begin{aligned}
& \left|\mu_{\Omega}\left(\left(b-b_{Q_{j}}\right) f_{j}\right)(x)\right| \\
\geq & \frac{C_{1}}{\left(\log \frac{2}{A_{1}}\right)^{\gamma}}\left\{\int_{0}^{\infty}\left(\int_{Q_{j}} \frac{\left(b(y)-b_{Q_{j}}\right) f_{j}(y)}{|x-y|^{n-1}} \chi_{\{|x-y| \leq t\}} d y\right)^{2} \frac{d t}{t^{3}}\right\}^{1 / 2} \\
\geq & C\left\{\int_{\left|x-y_{j}\right|}^{\infty}\left(\int_{Q_{j}} \frac{\left(b(y)-b_{Q_{j}}\right) f_{j}(y)}{|x-y|^{n-1}} \chi_{\{|x-y| \leq t\}} d y\right)^{2} \frac{d t}{t^{3}}\right\}^{1 / 2} \\
\geq & C\left|x-y_{j}\right| \int_{Q_{j}}|x-y|^{1-n}\left(b(y)-b_{Q_{j}}\right) f_{j}(y) \int_{\substack{\left|x-y_{j}\right| \leq t \\
|x-y| \leq t}} \frac{d t}{t^{3}} d y \\
\geq & C\left|x-y_{j}\right|^{-n} \int_{Q_{j}}\left(b(y)-b_{Q_{j}}\right) f_{j}(y) d y \\
= & C\left|x-y_{j}\right|^{-n}\left|Q_{j}\right|^{-1 / p+\lambda /(n p)} \int_{Q_{j}}\left|b(y)-b_{Q_{j}}\right| d y \\
\geq & C \zeta\left|Q_{j}\right|^{1 / p^{\prime}+\lambda /(n p)}\left|x-y_{j}\right|^{-n} .
\end{aligned}
$$

On the other hand, for $x \in\left(A_{2} Q_{j}\right)^{c}$, by $\Omega \in L^{\infty}\left(S^{n-1}\right)$, (2.2), (2.5), the Minkowski inequality and the Hölder inequality, we obtain

$$
\begin{aligned}
& \left|\mu_{\Omega}\left(\left(b-b_{Q_{j}}\right) f_{j}\right)(x)\right| \\
\leq & \int_{Q_{j}}\left|b(y)-b_{Q_{j}}\right|\left|f_{j}(y)\right| \frac{|\Omega(x-y)|}{|x-y|^{n}} d y \\
\leq & C\left|Q_{j}\right|^{1 / p^{\prime}}\left(\frac{1}{\left|Q_{j}\right|} \int_{Q_{j}}\left|b(y)-b_{Q_{j}}\right|^{p^{\prime}} d y\right)^{\frac{1}{p^{\prime}}}\left(\int_{Q_{j}} \frac{\left|f_{j}(y)\right|^{p}}{|x-y|^{p n}} d y\right)^{\frac{1}{p}} \\
\leq & C\left|Q_{j}\right|^{1 / p^{\prime}}\left(\int_{Q_{j}}\left|f_{j}(y)\right|^{p}|x-y|^{-p n} d y\right)^{1 / p} \\
\leq & C\left|Q_{j}\right|^{1 / p^{\prime}+\lambda /(n p)}\left|x-y_{j}\right|^{-n} .
\end{aligned}
$$

By (2.4) and Lemma 2.5, we have 


$$
\begin{aligned}
& \left|\left(b(x)-b_{Q_{j}}\right) \mu_{\Omega}\left(f_{j}\right)(x)\right| \\
& =\left|b(x)-b_{Q_{j}}\right|\left\{\int_{0}^{\infty} \mid \int_{\mathbb{R}^{n}} f_{j}(y)\left(\frac{\Omega(x-y)}{|x-y|^{n-1}} \chi_{\{|x-y| \leq t\}}\right.\right. \\
& \left.\left.-\frac{\Omega\left(x-y_{j}\right)}{\left|x-y_{j}\right|^{n-1}} \chi_{\left\{\left|x-y_{j}\right| \leq t\right\}}\right)\left.d y\right|^{2} \frac{d t}{t^{3}}\right\}^{\frac{1}{2}} \\
& \leq\left|b(x)-b_{Q_{j}}\right|\left\{\left(\int_{0}^{\infty}\left(\int_{|x-y| \leq t<\left|x-y_{j}\right|} \frac{|\Omega(x-y)|}{|x-y|^{n-1}}\left|f_{j}(y)\right| d y\right)^{2} \frac{d t}{t^{3}}\right)^{\frac{1}{2}}\right. \\
& +\left(\int_{0}^{\infty}\left(\int_{\left|x-y_{j}\right| \leq t<|x-y|} \frac{\left|\Omega\left(x-y_{j}\right)\right|}{\left|x-y_{j}\right|^{n-1}}\left|f_{j}(y)\right| d y\right)^{2} \frac{d t}{t^{3}}\right)^{\frac{1}{2}} \\
& \left.+\left(\int_{0}^{\infty}\left(\int_{\substack{\left|x-y_{j}\right| \leq t \\
|x-y| \leq t}}\left|\frac{\Omega(x-y)}{|x-y|^{n-1}}-\frac{\Omega\left(x-y_{j}\right)}{\left|x-y_{j}\right|^{n-1}}\right|\left|f_{j}(y)\right| d y\right)^{2} \frac{d t}{t^{3}}\right)^{\frac{1}{2}}\right\} \\
& \leq\left|b(x)-b_{Q_{j}}\right| \int_{Q_{j}} \frac{|\Omega(x-y)|}{|x-y|^{n-1} \mid} f_{j}(y) \mid\left(\int_{\substack{|x-y| \leq t \\
\left|x-y_{j}\right|>t}} \frac{d t}{t^{3}}\right)^{1 / 2} d y \\
& +\left|b(x)-b_{Q_{j}}\right| \int_{Q_{j}} \frac{\left|\Omega\left(x-y_{j}\right)\right|}{\left|x-y_{j}\right|^{n-1} \mid} f_{j}(y) \mid\left(\int_{\substack{|x-y|>t \\
\left|x-y_{j}\right| \leq t}} \frac{d t}{t^{3}}\right)^{1 / 2} d y \\
& +\left|b(x)-b_{Q_{j}}\right| \int_{Q_{j}}\left|f_{j}(y)\right|\left|\frac{\Omega(x-y)}{|x-y|^{n-1}}-\frac{\Omega\left(x-y_{j}\right)}{\left|x-y_{j}\right|^{n-1} \mid}\right|\left(\int_{\substack{|x-y| \leq t \\
\left|x-y_{j}\right| \leq t}} \frac{d t}{t^{3}}\right)^{1 / 2} d y \\
& \leq C\left|b(x)-b_{Q_{j}}\right|\left(\int_{Q_{j}} \frac{\left|f_{j}(y)\right|}{\left|x-y_{j}\right|^{n}\left(\log \frac{2\left|x-y_{j}\right|}{q_{j}}\right)^{\gamma}} d y+q_{j}^{1 / 2} \int_{Q_{j}} \frac{\left|f_{j}(y)\right|}{\left|x-y_{j}\right|^{n+1 / 2}} d y\right) \\
& \leq C\left|Q_{j}\right|^{1 / p^{\prime}+\lambda /(n p)} \frac{\left|b(x)-b_{Q_{j}}\right|^{q_{j}}}{\left|x-y_{j}\right|^{n}\left(\log \frac{2\left|x-y_{j}\right|}{q_{j}}\right)^{\gamma}} .
\end{aligned}
$$

Since $\left|b_{2 Q}-b_{Q}\right| \leq C\|b\|_{*}=C$ by $\|b\|_{*}=1$, we have

$$
\left(\int_{2^{s} q_{j}<\left|x-y_{j}\right| \leq 2^{s+1} q_{j}}\left|b(x)-b_{Q_{j}}\right|^{p} d x\right)^{\frac{1}{p}} \leq C 2^{s n / p} s\left|Q_{j}\right|^{1 / p} .
$$

For $v>A_{2}$, using (2.11) and the above inequality, we obtain

$$
\begin{aligned}
& \left(\int_{\left|x-y_{j}\right|>v q_{j}}\left|\left(b(x)-b_{Q_{j}}\right) \mu_{\Omega}\left(f_{j}\right)(x)\right|^{p} d x\right)^{\frac{1}{p}} \\
\leq & C\left|Q_{j}\right|^{1 / p^{\prime}+\lambda /(n p)}\left(\int_{\left|x-y_{j}\right|>v q_{j}} \frac{\left|b(x)-b_{Q_{j}}\right|^{p}}{\left|x-y_{j}\right|^{n p}\left(\log \frac{2\left|x-y_{j}\right|}{q_{j}}\right)^{\gamma p}} d x\right)^{\frac{1}{p}} \\
\leq & C\left|Q_{j}\right|^{1 / p^{\prime}+\lambda /(n p)} \sum_{s=\left[\log _{2} v\right]}^{\infty}\left(\int_{2^{s} q_{j}<\left|x-y_{j}\right| \leq 2^{s+1} q_{j}} \frac{\left|b(x)-b_{Q_{j}}\right|^{p}}{\left|x-y_{j}\right|^{n p}\left(\log \frac{2\left|x-y_{j}\right|}{q_{j}}\right)^{\gamma p}} d x\right)^{\frac{1}{p}} \\
\leq & C\left|Q_{j}\right|^{\lambda /(n p)} \sum_{s=\left[\log _{2} v\right]}^{\infty} 2^{-s(n-n / p)} s^{1-\gamma} \\
\leq & C\left|Q_{j}\right|^{\lambda /(n p)}(\log v)^{1-\gamma} v^{-n(1-1 / p)} .
\end{aligned}
$$


Then for $u>v>A_{2}$, using (2.9) and (2.12) we get

$$
\begin{aligned}
& \left(\int_{\left\{v q_{j}<\left|x-y_{j}\right| \leq u q_{j}\right\}}\left|\left[b, \mu_{\Omega}\right] f_{j}(x)\right|^{p} d x\right)^{\frac{1}{p}} \\
\geq & \left(\int_{\left\{v q_{j}<\left|x-y_{j}\right| \leq u q_{j}\right\} \cap\left\{x:\left(x-y_{j}\right)^{\prime} \in \Lambda\right\}}\left|\mu_{\Omega}\left(\left(b-b_{Q_{j}}\right) f_{j}\right)(x)\right|^{p} d x\right)^{\frac{1}{p}} \\
& -\left(\int_{\left|x-y_{j}\right|>v q_{j}}\left|\left(b(x)-b_{Q_{j}}\right) \mu_{\Omega}\left(f_{j}\right)(x)\right|^{p} d x\right)^{\frac{1}{p}} \\
\geq & C \zeta\left|Q_{j}\right|^{1 / p^{\prime}+\lambda /(n p)}\left(\int_{\left\{v q_{j}<\left|x-y_{j}\right| \leq u q_{j}\right\} \cap\left\{x:\left(x-y_{j}\right)^{\prime} \in \Lambda\right\}} \frac{1}{\left|x-y_{j}\right|^{p n}} d x\right)^{\frac{1}{p}} \\
& -C\left|Q_{j}\right|^{\lambda /(n p)}(\log v)^{1-\gamma} v^{-n(1-1 / p)} \\
\geq & A_{3} \zeta\left|Q_{j}\right|^{\lambda /(n p)}\left(v^{-n p+n}-u^{-n p+n}\right)^{1 / p}-A_{4}\left|Q_{j}\right|^{\lambda /(n p)}(\log v)^{1-\gamma} v^{-n+n / p}
\end{aligned}
$$

From (2.10) and (2.12), it follows that

$$
\begin{aligned}
& \left(\int_{\left|x-y_{j}\right|>u q_{j}}\left|\left[b, \mu_{\Omega}\right] f_{j}(x)\right|^{p} d x\right)^{\frac{1}{p}} \\
\leq & \left(\int_{\left|x-y_{j}\right|>u q_{j}}\left|\mu_{\Omega}\left(\left(b-b_{Q_{j}}\right) f_{j}\right)(x)\right|^{p} d x\right)^{\frac{1}{p}} \\
& +\left(\int_{\left|x-y_{j}\right|>u q_{j}}\left|\left(b(x)-b_{Q_{j}}\right) \mu_{\Omega}\left(f_{j}\right)(x)\right|^{p} d x\right)^{\frac{1}{p}} \\
\leq & A_{5}\left|Q_{j}\right|^{\lambda /(n p)} u^{-n+n / p}+A_{6}\left|Q_{j}\right|^{\lambda /(n p)}(\log u)^{1-\gamma} u^{-n+n / p} .
\end{aligned}
$$

By (2.13) and (2.14), there exist $A_{7}, B=B\left(\Omega, p, n, \lambda, \zeta, A_{3}, A_{4}, A_{5}, A_{6}\right)>1$ and $A_{9}$ such that $A_{2}<A_{7}$,

$$
\left(\int_{A_{7} q_{j}<\left|x-y_{j}\right| \leq B A_{7} q_{j}}\left|\left[b, \mu_{\Omega}\right] f_{j}(y)\right|^{p} d y\right)^{1 / p} \geq A_{9}\left|Q_{j}\right|^{\lambda /(n p)}
$$

and

$$
\left(\int_{\left|x-y_{j}\right|>B A_{7} q_{j}}\left|\left[b, \mu_{\Omega}\right] f_{j}(y)\right|^{p} d y\right)^{1 / p} \leq \frac{A_{9}}{4}\left|Q_{j}\right|^{\lambda /(n p)} .
$$

Let $A_{8}=B A_{7}$ and let $E \subset\left\{x: A_{7} q_{j}<\left|x-y_{j}\right|<A_{8} q_{j}\right\}$ be an arbitrary 
measurable set. Then by (2.10) and (2.11), we have

$$
\begin{aligned}
& \left(\int_{E}\left|\left[b, \mu_{\Omega}\right] f_{j}(x)\right|^{p} d x\right)^{\frac{1}{p}} \\
\leq & \left(\int_{E}\left|\mu_{\Omega}\left(\left(b-b_{Q_{j}}\right) f_{j}\right)(x)\right|^{p} d x\right)^{\frac{1}{p}} \\
& +\left(\int_{E}\left|\left(b(x)-b_{Q_{j}}\right) \mu_{\Omega}\left(f_{j}\right)(x)\right|^{p} d x\right)^{\frac{1}{p}} \\
\leq & C\left|Q_{j}\right|^{1 / p^{\prime}+\lambda /(n p)}\left(\int_{E}\left|x-y_{j}\right|^{-p n} d x\right)^{\frac{1}{p}} \\
& +C\left|Q_{j}\right|^{1 / p^{\prime}+\lambda /(n p)}\left(\int_{E} \frac{\left|b(x)-b_{Q_{j}}\right|^{p}}{\left|x-y_{j}\right|^{n p}\left(\log \frac{2\left|x-y_{j}\right|}{q_{j}}\right)^{\gamma p}} d x\right)^{\frac{1}{p}} \\
\leq & A_{10}\left|Q_{j}\right|^{\lambda /(n p)}\left\{\frac{|E|^{1 / p}}{\left|Q_{j}\right|^{1 / p}}+\left(\frac{1}{\left|Q_{j}\right|} \int_{E}\left|b(x)-b_{Q_{j}}\right|^{p} d x\right)^{\frac{1}{p}}\right\} .
\end{aligned}
$$

Let $h_{j}(x)=b(x)-b_{Q_{j}}$, and for $0<\omega<\infty$, denote by $\lambda_{h_{j}}(\omega)$ the measure of the following set:

$$
\left\{A_{7} q_{j}<\left|x-y_{j}\right|<A_{8} q_{j}:\left|h_{j}(x)\right|>\omega\right\} .
$$

Then by Lemma 2.1, there exist positive constants $A_{11}, A_{12}$ and $A_{13}$, such that

$$
\lambda_{h_{j}}\left(\omega+A_{11}\right) \leq A_{12}\left|Q_{j}\right| e^{-A_{13} \omega} .
$$

Hence, $\lambda_{h_{j}}(\omega) \leq A_{12}\left|Q_{j}\right| e^{-A_{13}\left(\omega-A_{11}\right)}$. For $t>0$, let $h_{j}^{*}(t)=\inf \left\{\omega: \lambda_{h_{j}}(\omega) \leq\right.$ $t\}$. Then when $0<t<A_{12}\left|Q_{j}\right|$,

$$
h_{j}^{*}(t) \leq \frac{1}{A_{13}} \log \frac{A_{12}\left|Q_{j}\right|}{t}+A_{11} .
$$

Recall $E \subset\left\{x: A_{7} q_{j}<\left|x-y_{j}\right|<A_{8} q_{j}\right\}$, applying Lemma 2.2 and (2.18), if $|E| \ll A_{12}\left|Q_{j}\right|$, we have

$$
\begin{aligned}
\frac{1}{\left|Q_{j}\right|} \int_{E}\left|b(x)-b_{Q_{j}}\right|^{p} d x & \leq \frac{1}{\left|Q_{j}\right|} \int_{0}^{|E|}\left|h_{j}^{*}(t)\right|^{p} d t \\
& \leq \frac{1}{\left|Q_{j}\right|} \int_{0}^{|E|}\left(A_{11}-\frac{1}{A_{13}} \log \frac{t}{A_{12}\left|Q_{j}\right|}\right)^{p} d t \\
& =A_{12} \int_{0}^{|E| /\left(A_{12}\left|Q_{j}\right|\right)}\left(A_{11}-\frac{1}{A_{13}} \log t\right)^{p} d t \\
& \leq A_{14} \frac{|E|}{\left|Q_{j}\right|}\left(1+\log \frac{A_{12}\left|Q_{j}\right|}{|E|}\right)^{[p]+1} .
\end{aligned}
$$


Combining (2.17) with (2.19), there exists a positive constant $A_{15}<\min \left\{A_{12}^{1 / n}, A_{8}\right\}$, such that

$$
\left(\int_{E}\left|\left[b, \mu_{\Omega}\right] f_{j}(y)\right|^{p} d y\right)^{\frac{1}{p}} \leq \frac{A_{9}}{4}\left|Q_{j}\right|^{\lambda /(n p)}
$$

for every measurable set $E$ satisfying $E \subset\left\{x: A_{7} q_{j}<\left|x-y_{j}\right|<A_{8} q_{j}\right\}$ and $|E| /\left|Q_{j}\right|<A_{15}^{n}$. Now we choose a subsequence $\left\{Q_{j_{k}}\right\}$ satisfying

$$
q_{j_{k+1}} / q_{j_{k}}<A_{15} / A_{8}
$$

For $m>0$, we have

$$
\begin{aligned}
& \left(\int_{B\left(y_{j_{k}}, A_{8} q_{j_{k}}\right)}\left|\left[b, \mu_{\Omega}\right] f_{j_{k}}-\left[b, \mu_{\Omega}\right] f_{j_{k+m}}\right|^{p} d x\right)^{\frac{1}{p}} \\
\geq & \left(\int_{G_{1}}\left|\left[b, \mu_{\Omega}\right] f_{j_{k}}-\left[b, \mu_{\Omega}\right] f_{j_{k+m}}\right|^{p} d x\right)^{\frac{1}{p}} \\
\geq & \left(\int_{G_{1}}\left|\left[b, \mu_{\Omega}\right] f_{j_{k}}\right|^{p} d x\right)^{\frac{1}{p}}-\left(\int_{G_{2}}\left|\left[b, \mu_{\Omega}\right] f_{j_{k+m}}\right|^{p} d x\right)^{\frac{1}{p}},
\end{aligned}
$$

where

$$
\begin{aligned}
& G_{1}=\left\{x: A_{7} q_{j_{k}}<\left|x-y_{j_{k}}\right|<A_{8} q_{j_{k}}\right\} \backslash\left\{x:\left|x-y_{j_{k+m}}\right| \leq A_{8} q_{j_{k+m}}\right\} \subset B\left(y_{j_{k}}, A_{8} q_{j_{k}}\right) \\
& \text { and } G_{2}=\left\{x:\left|x-y_{j_{k+m}}\right|>A_{8} q_{j_{k+m}}\right\} \text {. Let } \\
& \qquad G=\left\{x: A_{7} q_{j_{k}}<\left|x-y_{j_{k}}\right|<A_{8} q_{j_{k}}\right\},
\end{aligned}
$$

then $G_{1}=G-\left(G_{2}^{c} \cap G\right)$. Thus by (2.15) and (2.16), we get

$$
\begin{aligned}
& \left(\int_{B\left(y_{j_{k}}, A_{8} q_{j_{k}}\right)}\left|\left[b, \mu_{\Omega}\right] f_{j_{k}}-\left[b, \mu_{\Omega}\right] f_{j_{k+m}}\right|^{p} d x\right)^{\frac{1}{p}} \\
\geq & \left(\int_{G}\left|\left[b, \mu_{\Omega}\right] f_{j_{k}}\right|^{p} d x-\int_{G_{2}^{c} \cap G}\left|\left[b, \mu_{\Omega}\right] f_{j_{k}}\right|^{p} d x\right)^{\frac{1}{p}}-\left(\int_{G_{2}}\left|\left[b, \mu_{\Omega}\right] f_{j_{k+m}}\right|^{p} d x\right)^{\frac{1}{p}} \\
\geq & \left(A_{9}^{p}\left|Q_{j_{k}}\right|^{\lambda / n}-\int_{G_{2}^{c} \cap G}\left|\left[b, \mu_{\Omega}\right] f_{j_{k}}\right|^{p} d x\right)^{\frac{1}{p}}-\frac{A_{9}}{4}\left|Q_{j_{k+m}}\right| \lambda /(n p) .
\end{aligned}
$$

Since $\left(G_{2}^{c} \cap G\right) \subset G$ and by (2.21), we have

$$
\frac{\left|G_{2}^{c} \cap G\right|}{\left|Q_{j_{k}}\right|} \leq \frac{A_{8}^{n} q_{j_{k+m}}^{n}}{q_{j_{k}}^{n}}<A_{8}^{n}\left(\frac{A_{15}^{n}}{A_{8}^{n}}\right)^{m}<A_{15}^{n} .
$$


By (2.20), we get

$$
\int_{G_{2}^{c} \cap G}\left|\left[b, \mu_{\Omega}\right] f_{j_{k}}\right|^{p} d x \leq\left(\frac{A_{9}}{4}\right)^{p}\left|Q_{j_{k}}\right|^{\lambda / n}
$$

So

$$
\left(\int_{B\left(y_{j_{k}}, A_{8} q_{j_{k}}\right)}\left|\left[b, \mu_{\Omega}\right] f_{j_{k}}-\left[b, \mu_{\Omega}\right] f_{j_{k+m}}\right|^{p} d x\right)^{\frac{1}{p}} \geq \frac{A_{9}}{4}\left|Q_{j_{k}}\right|^{\lambda /(n p)} .
$$

Then

$$
\left(\frac{1}{q_{j_{k}}^{\lambda}} \int_{B\left(y_{j_{k}}, A_{8} q_{j_{k}}\right)}\left|\left[b, \mu_{\Omega}\right] f_{j_{k}}-\left[b, \mu_{\Omega}\right] f_{j_{k+m}}\right|^{p} d x\right)^{\frac{1}{p}} \geq A_{16} .
$$

Therefore

$$
\left\|\left[b, \mu_{\Omega}\right] f_{j_{k}}-\left[b, \mu_{\Omega}\right] f_{j_{k+m}}\right\|_{p, \lambda} \geq A_{17} .
$$

Thus the sequence $\left\{\left[b, \mu_{\Omega}\right] f_{j_{k}}\right\}_{k=1}^{\infty}$ has no any convergence subsequence in $L^{p, \lambda}\left(\mathbb{R}^{n}\right)$, i.e., $\left[b, \mu_{\Omega}\right]$ is not a compact operator in $L^{p, \lambda}\left(\mathbb{R}^{n}\right)$. This contradiction shows that $b$ must satisfy the condition (i) of Lemma 2.3.

Similarly, we may show that if $b$ does not satisfy the conditions (ii) or (iii) in Lemma 2.3 , then $\left[b, \mu_{\Omega}\right]$ is also not a compact operator in $L^{p, \lambda}\left(\mathbb{R}^{n}\right)$. For simplicity, we give only the outline of the proofs. In fact, if $b$ does not satisfy the condition (ii) of Lemma 2.3, we can select a sequence $\left\{Q_{j}\right\}$ such that $(2.1)$ holds and $\lim _{j \rightarrow \infty} q_{j}=$ $\infty$, where $q_{j}$ is the diameters of $Q_{j}$, and $y_{j}$ is the center of $Q_{j}$. Similarly, we select a sequence $\left\{f_{j}\right\} \subset L^{p, \lambda}\left(\mathbb{R}^{n}\right)$ such that (2.15), (2.16) and (2.20) hold. Hence, if we choose a subsequence $\left\{Q_{j_{k}}\right\}$ such that $q_{j_{k}}>1$ and

$$
q_{j_{k}} / q_{j_{k+1}}<A_{15} / A_{8}
$$

then for $m>0$, we have

$$
\begin{aligned}
& \left(\int_{B\left(y_{j_{k+m}}, A_{8} q_{j_{k+m}}\right)}\left|\left[b, \mu_{\Omega}\right] f_{j_{k}}-\left[b, \mu_{\Omega}\right] f_{j_{k+m}}\right|^{p} d x\right)^{\frac{1}{p}} \\
\geq & \left(\int_{G_{1}}\left|\left[b, \mu_{\Omega}\right] f_{j_{k}}-\left[b, \mu_{\Omega}\right] f_{j_{k+m}}\right|^{p} d x\right)^{\frac{1}{p}} \\
\geq & \left(\left.\int_{G_{1}}\left|\left[b, \mu_{\Omega}\right] f_{j_{k+m}}\right|\right|^{p} d x\right)^{\frac{1}{p}}-\left(\int_{G_{2}}\left|\left[b, \mu_{\Omega}\right] f_{j_{k}}\right|^{p} d x\right)^{\frac{1}{p}},
\end{aligned}
$$

where

$$
\begin{aligned}
G_{1} & =\left\{x: A_{7} q_{j_{k+m}}<\left|x-y_{j_{k+m}}\right|<A_{8} q_{j_{k+m}}\right\} \backslash\left\{x:\left|x-y_{j_{k}}\right| \leq A_{8} q_{j_{k}}\right\} \\
& \subset B\left(y_{j_{k+m}}, A_{8} q_{j_{k+m}}\right)
\end{aligned}
$$


and $G_{2}=\left\{x:\left|x-y_{j_{k}}\right|>A_{8} q_{j_{k}}\right\}$. Set

$$
G=\left\{x: A_{7} q_{j_{k+m}}<\left|x-y_{j_{k+m}}\right|<A_{8} q_{j_{k+m}}\right\},
$$

then $G_{1}=G-\left(G_{2}^{c} \bigcap G\right)$. Thus by (2.15) and (2.16) we get

$$
\begin{aligned}
& \left(\int_{B\left(y_{j_{k+m}}, A_{8} q_{j_{k+m}}\right)}\left|\left[b, \mu_{\Omega}\right] f_{j_{k}}-\left[b, \mu_{\Omega}\right] f_{j_{k+m}}\right|^{p} d x\right)^{\frac{1}{p}} \\
\geq & \left(\int_{G}\left|\left[b, \mu_{\Omega}\right] f_{j_{k+m}}\right|^{p} d x-\int_{G_{2}^{c} \cap G}\left|\left[b, \mu_{\Omega}\right] f_{j_{k+m}}\right|^{p} d x\right)^{\frac{1}{p}}-\left(\int_{G_{2}}\left|\left[b, \mu_{\Omega}\right] f_{j_{k}}\right|^{p} d x\right)^{\frac{1}{p}} \\
\geq & \left(\left.A_{9}^{p}\left|Q_{j_{k+m}}\right|\right|^{\lambda / n}-\left.\int_{G_{2}^{c} \cap G}\left|\left[b, \mu_{\Omega}\right] f_{j_{k+m}}\right|\right|^{p} d x\right)^{\frac{1}{p}}-\frac{A_{9}}{4}\left|Q_{j_{k}}\right|^{\lambda /(n p)} .
\end{aligned}
$$

Since $G_{2}^{c} \bigcap G \subset G$, by (2.22) we have

$$
\frac{\left|G_{2}^{c} \cap G\right|}{\left|Q_{j_{k+m}}\right|} \leq \frac{A_{8}^{n} q_{j_{k}}^{n}}{q_{j_{k+m}}^{n}}<A_{8}^{n}\left(\frac{A_{15}^{n}}{A_{8}^{n}}\right)^{m}<A_{15}^{n} .
$$

Thus, by $(2.20)$ we get

$$
\int_{G_{2}^{c} \cap G}\left|\left[b, \mu_{\Omega}\right] f_{j_{k+m}}\right|^{p} d x \leq\left(\frac{A_{9}}{4}\right)^{p}\left|Q_{j_{k+m}}\right|^{\lambda / n} .
$$

Hence

$$
\left(\int_{B\left(y_{j_{k+m}}, A_{8} q_{j_{k+m}}\right)}\left|\left[b, \mu_{\Omega}\right] f_{j_{k}}-\left[b, \mu_{\Omega}\right] f_{j_{k+m}}\right|^{p} d x\right)^{\frac{1}{p}} \geq \frac{A_{9}}{4}\left|Q_{j_{k+m}}\right|^{\lambda /(n p)} .
$$

and

$$
\left\|\left[b, \mu_{\Omega}\right] f_{j_{k}}-\left[b, \mu_{\Omega}\right] f_{j_{k+m}}\right\|_{p, \lambda} \geq A_{18} .
$$

Thus $\left\{\left[b, \mu_{\Omega}\right] f_{j_{k}}\right\}_{k=1}^{\infty}$ has no any convergence subsequence in $L^{p, \lambda}\left(\mathbb{R}^{n}\right)$. But this is contrary to the assumption that $\left[b, \mu_{\Omega}\right]$ is a compact operator in $L^{p, \lambda}\left(\mathbb{R}^{n}\right)$. Hence, $b$ should satisfy the condition (ii) of Lemma 2.3.

Finally, if $b$ does not satisfy the condition (iii) of Lemma 2.3, then there exist a cube $Q$ and sequence $\left\{y_{j}\right\}$ with $\lim _{j \rightarrow \infty}\left|y_{j}\right|=\infty$ such that $(2.1)$ holds for $\left\{Q_{j}=\right.$ $\left.Q+y_{j}\right\}$. Let

$$
E_{j}=\left\{x \in \mathbb{R}^{n}:\left|x-y_{j}\right|<A_{8} q^{\prime}\right\},
$$

where $q^{\prime}$ is the diameter of $Q$. We select a sequence $\left\{f_{j}\right\} \subset L^{p, \lambda}\left(\mathbb{R}^{n}\right)$ such that $(2.15)$ and $(2.16)$ hold. Now, we choose a subsequence $\left\{E_{j_{k}}\right\}$ such that 


$$
E_{j_{k}} \bigcap E_{j_{l}}=\emptyset \text { for } \quad l \neq k .
$$

Then for $m>0$, we have

$$
\begin{aligned}
& \left(\int_{B\left(y_{j_{k}}, A_{8} q^{\prime}\right)}\left|\left[b, \mu_{\Omega}\right] f_{j_{k}}-\left[b, \mu_{\Omega}\right] f_{j_{k+m}}\right|^{p} d x\right)^{\frac{1}{p}} \\
\geq & \left(\int_{G_{1}}\left|\left[b, \mu_{\Omega}\right] f_{j_{k}}-\left[b, \mu_{\Omega}\right] f_{j_{k+m}}\right|^{p} d x\right)^{\frac{1}{p}} \\
\geq & \left(\int_{G_{1}}\left|\left[b, \mu_{\Omega}\right] f_{j_{k}}\right|^{p} d x\right)^{\frac{1}{p}}-\left(\int_{G_{2}}\left|\left[b, \mu_{\Omega}\right] f_{j_{k+m}}\right|^{p} d x\right)^{\frac{1}{p}},
\end{aligned}
$$

where

$$
\begin{aligned}
& \left.G_{1}=\left\{x: A_{7} q^{\prime}<\left|x-y_{j_{k}}\right|<A_{8} q^{\prime}\right\} \backslash\left\{x: \mid x-y_{j_{k+m}}\right) \mid \leq A_{8} q^{\prime}\right\} \subset B\left(y_{j_{k}}, A_{8} q^{\prime}\right) \\
& \text { and } G_{2}=\left\{x:\left|x-y_{j_{k+m}}\right|>A_{8} q^{\prime}\right\} \text {. Let } \\
& \qquad G=\left\{x: A_{7} q^{\prime}<\left|x-y_{j_{k}}\right|<A_{8} q^{\prime}\right\} \text {, }
\end{aligned}
$$

then $G_{1}=G-G_{2}^{c}=G$. Thus by $(2.15)$ and (2.16) we get

$$
\begin{aligned}
& \left(\int_{B\left(y_{j_{k}}, A_{8} q^{\prime}\right)}\left|\left[b, \mu_{\Omega}\right] f_{j_{k}}-\left[b, \mu_{\Omega}\right] f_{j_{k+m}}\right|^{p} d x\right)^{\frac{1}{p}} \\
\geq & \left(\int_{G}\left|\left[b, \mu_{\Omega}\right] f_{j_{k}}\right|^{p} d x\right)^{\frac{1}{p}}-\left(\int_{G_{2}}\left|\left[b, \mu_{\Omega}\right] f_{j_{k+m}}\right|^{p} d x\right)^{\frac{1}{p}} \\
\geq & A_{9}|Q|^{\lambda /(n p)}-\frac{A_{9}}{4}|Q|^{\lambda /(n p)} \geq \frac{A_{9}}{4}|Q|^{\lambda /(n p)} .
\end{aligned}
$$

Hence

$$
\left\|\left[b, \mu_{\Omega}\right] f_{j_{k}}-\left[b, \mu_{\Omega}\right] f_{j_{k+m}}\right\|_{p, \lambda} \geq A_{19} .
$$

This is inconsistent with the compactness of $\left[b, \mu_{\Omega}\right]$ in $L^{p, \lambda}\left(\mathbb{R}^{n}\right)$. So, $b$ satisfies also the condition (iii) of Lemma 2.3.

\section{Proof of Theorem 2}

First we give some lemmas, which will be used in the proof of Theorem 2.

Lemma 3.1. ([4]). Suppose that $1 \leq p<\infty$ and $0<\lambda<n$. If the subset $G$ in $L^{p, \lambda}\left(\mathbb{R}^{n}\right)$ satisfies the following conditions:

$$
\sup _{f \in G}\|f\|_{p, \lambda}<\infty
$$




$$
\begin{gathered}
\lim _{y \rightarrow 0}\|f(\cdot+y)-f(\cdot)\|_{p, \lambda}=0 \text { uniformly in } f \in G, \\
\lim _{\alpha \rightarrow \infty}\left\|f \chi_{E_{\alpha}}\right\|_{p, \lambda}=0 \quad \text { uniformly in } f \in G,
\end{gathered}
$$

where $E_{\alpha}=\left\{x \in \mathbb{R}^{n}:|x|>\alpha\right\}$, then $G$ is strongly pre-compact set in $L^{p, \lambda}\left(\mathbb{R}^{n}\right)$.

Lemma 3.2. ([9]) Suppose that $0 \leq \beta<n, \Omega$ satisfies (1.1) and the $L^{q}$-Dini condition

$$
\int_{0}^{1} \frac{\omega_{q}(\delta)}{\delta} d \delta<\infty
$$

for $q \geq 1$. If there exists a positive constant $0<\theta<1 / 2$ such that $|x|<\theta R$, then we have the following inequality

$$
\begin{aligned}
& \left(\int_{R<|y|<2 R}\left|\frac{\Omega(y-x)}{|y-x|^{n-\beta}}-\frac{\Omega(y)}{|y|^{n-\beta}}\right|^{q} d y\right)^{1 / q} \\
\leq & C R^{n / q-(n-\beta)}\left\{\frac{|x|}{R}+\int_{|x| / 2 R}^{|x| / R} \frac{\omega_{q}(\delta)}{\delta} d \delta\right\},
\end{aligned}
$$

where the constant $C>0$ is independent of $R$ and $x$.

Lemma 3.3. ([12]). Suppose that $1<p<\infty$ and $1 \leq r<p<\infty$, then the maximal operator $\mathcal{M}_{r}$ and Calderon-Zygmund singular integral operator $T$ are bounded operators on $L^{p, \lambda}\left(\mathbb{R}^{n}\right)$, where $\mathcal{M}_{r} f(x)=\left\{\mathcal{M}\left(|f|^{r}\right)(x)\right\}^{1 / r}$ and $\mathcal{M}$ is the Hardy-Littlewood maximal operator.

Now let us return to the proof of Theorem 2. Suppose that $F$ is an arbitrary bounded set in $L^{p, \lambda}\left(\mathbb{R}^{n}\right)$, that is, there exists a constant $D>0$ such that $\|f\|_{p, \lambda} \leq$ $D$ for every $f \in F$. Let $G=\left\{\left[b, \mu_{\Omega}\right] f: f \in F\right\}$ if $b \in C_{c}^{\infty}\left(\mathbb{R}^{n}\right)$ and $\widetilde{G}=$ $\left\{\left[b, \mu_{\Omega}\right] f: f \in F\right\}$ if $b \in \operatorname{VMO}\left(\mathbb{R}^{n}\right)$. For $b \in \operatorname{VMO}\left(\mathbb{R}^{n}\right)$, by (2.7), we can easily get $\left[b, \mu_{\Omega}\right]$ is continuous in $L^{p, \lambda}\left(\mathbb{R}^{n}\right)$. So, by the definition of the compact operator (see [2], for example), it suffices to prove that for any bounded set $F$ in $L^{p, \lambda}\left(\mathbb{R}^{n}\right), \widetilde{G}$ is strongly pre-compact in $L^{p, \lambda}\left(\mathbb{R}^{n}\right)$. We first show that if (3.1)-(3.3) hold uniformly in G, then (3.1)-(3.3) hold also uniformly in $\widetilde{G}$ and thus $\left[b, \mu_{\Omega}\right]$ is a compact operator in $L^{p, \lambda}\left(\mathbb{R}^{n}\right)$.

In fact, suppose that $b \in \operatorname{VMO}\left(\mathbb{R}^{n}\right)$, then for any $\varepsilon>0$ there exists $b^{\varepsilon} \in$ $C_{c}^{\infty}\left(\mathbb{R}^{n}\right)$ such that $\left\|b-b^{\varepsilon}\right\|_{*}<\varepsilon$. By

$$
\begin{aligned}
& \left|\left[b, \mu_{\Omega}\right] f(x)-\left[b^{\varepsilon}, \mu_{\Omega}\right] f(x)\right| \\
\leq & \left\{\int_{0}^{\infty}\left|\int_{|x-y| \leq t} \frac{\Omega(x-y)}{|x-y|^{n-1}}\left[\left(b-b^{\varepsilon}\right)(x)-\left(b-b^{\varepsilon}\right)(y)\right] f(y) d y\right|^{2} \frac{d t}{t^{3}}\right\}^{1 / 2}
\end{aligned}
$$


and (2.7), we obtain

$$
\left\|\left[b, \mu_{\Omega}\right]-\left[b^{\varepsilon}, \mu_{\Omega}\right]\right\|_{L^{p, \lambda} \rightarrow L^{p, \lambda}} \leq\left\|\left[b-b^{\varepsilon}, \mu_{\Omega}\right]\right\|_{L^{p, \lambda} \rightarrow L^{p, \lambda}} \leq C \varepsilon .
$$

For any $f \in F$, by (3.1) and (3.5) we get

$$
\sup _{f \in F}\left\|\left[b, \mu_{\Omega}\right] f\right\|_{p, \lambda} \leq \sup _{f \in F}\left\|\left[b^{\varepsilon}, \mu_{\Omega}\right] f\right\|_{p, \lambda}+C D \varepsilon<\infty .
$$

On the other hand, by (3.2) and (3.5), for any $f \in F$

$$
\begin{aligned}
& \lim _{|y| \rightarrow 0}\left\|\left[b, \mu_{\Omega}\right] f(\cdot+y)-\left[b, \mu_{\Omega}\right] f(\cdot)\right\|_{p, \lambda} \\
\leq & \lim _{|y| \rightarrow 0}\left\|\left[b^{\varepsilon}, \mu_{\Omega}\right] f(\cdot+y)-\left[b^{\varepsilon}, \mu_{\Omega}\right] f(\cdot)\right\|_{p, \lambda}+2\left\|\left[b-b^{\varepsilon}, \mu_{\Omega}\right] f\right\|_{p, \lambda} \\
< & 2 C D \varepsilon .
\end{aligned}
$$

Therefore (3.2) holds uniformly for $\widetilde{G}$. Similarly, by (3.3) and (3.5), we see that $\lim _{\alpha \rightarrow+\infty}\left\|\left[b, \mu_{\Omega}\right] f \chi_{E_{\alpha}}\right\|_{p, \lambda} \leq \lim _{\alpha \rightarrow+\infty}\left\|\left[b^{\varepsilon}, \mu_{\Omega}\right] f \chi_{E_{\alpha}}\right\|_{p, \lambda}+\left\|\left[b-b^{\varepsilon}, \mu_{\Omega}\right] f\right\|_{p, \lambda} \leq C D \varepsilon$.

Thus (3.3) holds also for $\widetilde{G}$ uniformly. Therefore, by Lemma 3.1, we know $\widetilde{G}$ is a strongly pre-compact set in $L^{p, \lambda}\left(\mathbb{R}^{n}\right)$ and then $\left[b, \mu_{\Omega}\right]$ is a compact operator in $L^{p, \lambda}\left(\mathbb{R}^{n}\right)$.

Thus, it suffices to prove that (3.1)-(3.3) hold uniformly in $G$. Recalling $\|f\|_{p, \lambda} \leq D$ for every $f \in F$, and noticing that $b \in C_{c}^{\infty}\left(\mathbb{R}^{n}\right)$, by (2.7), we have

$$
\sup _{f \in F}\left\|\left[b, \mu_{\Omega}\right] f\right\|_{p, \lambda} \leq C\|b\|_{*} \sup _{f \in F}\|f\|_{p, \lambda} \leq C D\|b\|_{*}<\infty .
$$

Suppose that $\operatorname{supp} b \subset\{x:|x| \leq \beta\}$. For any $0<\varepsilon<1$, we take $\alpha>\max \{1, \beta\}$ such that $(\alpha-\beta)^{n(1-q)}<\varepsilon^{q}$. If $q \leq p$, then for any $x$ satisfying $|x|>\alpha$ and every $f \in F$, we have

$$
\begin{aligned}
\left|\left[b, \mu_{\Omega}\right] f(x)\right| & =\left\{\int_{0}^{\infty}\left|\int_{|x-y| \leq t} \frac{\Omega(x-y)}{|x-y|^{n-1}}(b(x)-b(y)) f(y) d y\right|^{2} \frac{d t}{t^{3}}\right\}^{1 / 2} \\
& \leq C \int_{|y| \leq \beta} \frac{|b(y)||\Omega(x-y)|}{|x-y|^{n-1}}|f(y)|\left\{\int_{|x-y| \leq t} \frac{d t}{t^{3}}\right\}^{1 / 2} d y \\
& \leq C \int_{|y| \leq \beta} \frac{|\Omega(x-y)|}{|x-y|^{n}}|f(y)| d y \\
& \leq C\left(\int_{|y| \leq \beta} \frac{|\Omega(x-y)|^{q}}{|x-y|^{q n}}|f(y)|^{q} d y\right)^{1 / q} .
\end{aligned}
$$


For every $s \in \mathbb{R}^{n}$ and $r>0$, by the Minkowski inequality and the choice of $\alpha$, we get

$$
\begin{aligned}
& \left(\frac{1}{r^{\lambda}} \int_{B(s, r)}\left|\left[b, \mu_{\Omega}\right] f(x)\right|^{p} \chi_{E_{\alpha}}(x) d x\right)^{1 / p} \\
\leq & C\|f\|_{p, \lambda}\left(\int_{|y|>\alpha-\beta} \frac{|\Omega(y)|^{q}}{|y|^{n q}} d y\right)^{1 / q} \\
\leq & C\|f\|_{p, \lambda}\left(\int_{\alpha-\beta}^{\infty} \int_{S^{n-1}}\left|\Omega\left(y^{\prime}\right)\right|^{q} d \sigma\left(y^{\prime}\right) \frac{d r}{r^{n q-n+1}}\right)^{1 / q} \\
\leq & C D\|\Omega\|_{L^{q}\left(S^{n-1}\right)} \varepsilon \leq C D \varepsilon .
\end{aligned}
$$

If $q>p$, we choose $q_{0}$ such that $1<q_{0} \leq p<q$. Notice that $\Omega \in L^{q_{0}}\left(S^{n-1}\right)$ and $\|\Omega\|_{L^{q_{0}\left(S^{n-1}\right)}} \leq C\|\Omega\|_{L^{q}\left(S^{n-1}\right)}$, by (3.7), for every $s \in \mathbb{R}^{n}$ and $r>0$, we still get

$$
\left(\frac{1}{r^{\lambda}} \int_{B(s, r)}\left|\left[b, \mu_{\Omega}\right] f(x)\right|^{p} \chi_{E_{\alpha}}(x) d x\right)^{1 / p} \leq C D\|\Omega\|_{L^{q_{0}\left(S^{n-1}\right)}} \varepsilon \leq C D \varepsilon
$$

The estimates (3.7) and (3.8) show that (3.3) holds for the commutator $\left[b, \mu_{\Omega}\right]$ in $G$ uniformly. Finally, to finish the proof of Theorem 2, it remains to show (3.2) holds for the commutator $\left[b, \mu_{\Omega}\right]$ in $G$ uniformly. We need to prove that for any $\varepsilon>0$, if $|z|$ is sufficiently small depended only on $\varepsilon$, then for every $f \in F$,

$$
\left\|\left[b, \mu_{\Omega}\right] f(\cdot+z)-\left[b, \mu_{\Omega}\right] f(\cdot) \mid\right\|_{p, \lambda} \leq C \varepsilon .
$$

Then for $z \in \mathbb{R}^{n}$, we write

$$
\begin{aligned}
&\left|\left[b, \mu_{\Omega}\right] f(x+z)-\left[b, \mu_{\Omega}\right] f(x)\right| \\
& \leq\left\{\int_{0}^{\infty} \mid \int_{|x-y| \leq t} \frac{\Omega(x-y)}{|x-y|^{n-1}}(b(x)-b(y)) f(y) d y\right. \\
&\left.-\left.\int_{|x+z-y| \leq t} \frac{\Omega(x+z-y)}{|x+z-y|^{n-1}}(b(x+z)-b(y)) f(y) d y\right|^{2} \frac{d t}{t^{3}}\right\}^{1 / 2} \\
&:=\left\{\int_{0}^{\infty}|I(x, t)|^{2} \frac{d t}{t^{3}}\right\}^{1 / 2} .
\end{aligned}
$$

We take $\varepsilon$ such that $0<\varepsilon<\frac{1}{2}$. Then for $z \in \mathbb{R}^{n}$, decompose $I(x, t)$ as 


$$
\begin{aligned}
& I(x, t) \\
& =\int_{|x-y|>e^{\frac{1}{\varepsilon}|z|,|x-y| \leq t}|x+z-y|>t} \frac{\Omega(x-y)}{|x-y|^{n-1}}(b(x+z)-b(y)) f(y) d y
\end{aligned}
$$

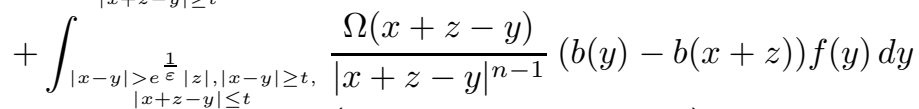

$$
\begin{aligned}
& +\int_{|x-y|>e^{\frac{1}{\varepsilon}|z|,|x-y| \leq t}|x+z-y| \leq t}\left(\frac{\Omega(x-y)}{|x-y|^{n-1}}-\frac{\Omega(x+z-y)}{|x+z-y|^{n-1}}\right)(b(x+z)-b(y)) f(y) d y \\
& +\int_{|x-y|>e^{\frac{1}{\varepsilon}}|z|,|x-y| \leq t} \frac{\Omega(x-y)}{|x-y|^{n-1}}(b(x)-b(x+z)) f(y) d y \\
& +\int_{|x-y| \leq e^{\frac{1}{\varepsilon}}|z|,|x-y| \leq t} \frac{\Omega(x-y)}{|x-y|^{n-1}}(b(x)-b(y)) f(y) d y \\
& +\int_{\substack{|x-y| \leq e \\
|x+z-y|<t}} \frac{\Omega(z|z| z-y)}{|x+z-y|^{n-1}}(b(y)-b(x+z)) f(y) d y \\
& :=J_{1}(x, t)+J_{2}(x, t)+J_{3}(x, t)+J_{4}(x, t)+J_{5}(x, t)+J_{6}(x, t) .
\end{aligned}
$$

By $|b(x+z)-b(y)|<C$ and the Minkowski inequality, we have

$$
\begin{aligned}
& \left(\int_{0}^{\infty}\left|J_{1}(x, t)\right|^{2} \frac{d t}{t^{3}}\right)^{1 / 2} \\
& =\left(\int_{0}^{\infty} \mid \int_{\substack{|x-y| \leq t,|x+z-y| \geq \frac{1}{\varepsilon}|z| \geq t \\
|x-y|^{n-1}}} \frac{\Omega(x-y)}{|f(y)||\Omega(x-y)|}\left\{\int_{\substack{|x-y| \leq t \\
|x+z-y| \geq t}} \frac{d t}{t^{3}}\right\}^{1 / 2} d y\right. \\
& \left.\leq\left. C \int_{|x-y|>e^{\frac{1}{\varepsilon}|z|}} \frac{|x-y|^{n-1}}{\mid x-y)} d y\right|^{2} \frac{d t}{t^{3}}\right)^{1 / 2} \\
& \leq C \int_{|x-y|>e^{\frac{1}{\varepsilon}}|z|} \frac{|z|^{1 / 2}|\Omega(x-y)|}{|x-y|^{n+1 / 2}}|f(y)| d y .
\end{aligned}
$$

Since $\Omega \in L^{1}\left(S^{n-1}\right)$, for every $s \in \mathbb{R}^{n}$ and $r>0$ we get

$$
\begin{aligned}
& \left\{\frac{1}{r^{\lambda}} \int_{B(s, r)}\left(\int_{0}^{\infty}\left|J_{1}(x, t)\right|^{2} \frac{d t}{t^{3}}\right)^{p / 2} d x\right\}^{\frac{1}{p}} \\
\leq & C\left\{\frac{1}{r^{\lambda}} \int_{B(s, r)}\left(\int_{|y|>e^{\frac{1}{\varepsilon}}|z|} \frac{|z|^{1 / 2}|\Omega(y)|}{|y|^{n+1 / 2}}|f(x-y)| d y\right)^{p} d x\right\}^{1 / p} \\
\leq & C\|f\|_{p, \lambda} \int_{|y|>e^{\frac{1}{\varepsilon}}|z|} \frac{|z|^{1 / 2}|\Omega(y)|}{|y|^{n+1 / 2}} d y \\
= & C\|f\|_{p, \lambda}|z|^{1 / 2} \int_{e^{\frac{1}{\varepsilon}}|z|}^{\infty} \frac{d r}{r^{1+1 / 2}} \int_{S^{n-1}}\left|\Omega\left(y^{\prime}\right)\right| d \sigma\left(y^{\prime}\right) \\
\leq & C e^{-\frac{1}{2 \varepsilon}}\|f\|_{p, \lambda} \\
\leq & C D \varepsilon .
\end{aligned}
$$

\section{Hence}




$$
\left\|\left\{\int_{0}^{\infty}\left|J_{1}(\cdot, t)\right|^{2} \frac{d t}{t^{3}}\right\}^{1 / 2}\right\|_{p, \lambda} \leq C D \varepsilon
$$

Similar to the estimate of $J_{1}(x, t)$, we can get

$$
\left\{\int_{0}^{\infty}\left|J_{2}(x, t)\right|^{2} \frac{d t}{t^{3}}\right\}^{1 / 2} \leq C \int_{|x-y|>e^{\frac{1}{\varepsilon}}|z|} \frac{|z|^{1 / 2}|\Omega(x+z-y)|}{|x+z-y|^{n+1 / 2}}|f(y)| d y .
$$

Thus for any $s \in \mathbb{R}^{n}$ and $r>0$, we have

$$
\begin{aligned}
& \left\{\frac{1}{r^{\lambda}} \int_{B(s, r)}\left(\int_{0}^{\infty}\left|J_{2}(x, t)\right|^{2} \frac{d t}{t^{3}}\right)^{p / 2} d x\right\}^{\frac{1}{p}} \\
& \leq C\left\{\frac{1}{r^{\lambda}} \int_{B(s, r)}\left(\int_{|y|>\left(e^{\frac{1}{\varepsilon}}-1\right)|z|} \frac{|z|^{1 / 2}|\Omega(y)|}{|y|^{n+1 / 2}}|f(x+z-y)| d y\right)^{p} d x\right\}^{1 / p} \\
& \leq C\|f\|_{p, \lambda}|z|^{1 / 2} \int_{|y|>\left(e^{\frac{1}{\varepsilon}}-1\right)|z|} \frac{|\Omega(y)|}{|y|^{n+1 / 2}} d y \\
& \leq C\left(e^{\frac{1}{\varepsilon}}-1\right)^{-1 / 2}\|f\|_{p, \lambda} \\
& \leq C D \varepsilon
\end{aligned}
$$

\section{Therefore}

$$
\left\|\left\{\int_{0}^{\infty}\left|J_{2}(\cdot, t)\right|^{2} \frac{d t}{t^{3}}\right\}^{1 / 2}\right\|_{p, \lambda} \leq C D \varepsilon
$$

About $J_{3}$. By the Minkowski inequality and $|b(x+z)-b(y)|<C$, we have

$$
\begin{aligned}
& \left\{\int_{0}^{\infty}\left|J_{3}(x, t)\right|^{2} \frac{d t}{t^{3}}\right\}^{1 / 2} \\
= & \left\{\int_{0}^{\infty} \mid \int_{\substack{|x-y|>e^{\frac{1}{\varepsilon}}|z|,|x-y| \leq t \\
|x+z-y| \leq t}}\left(\frac{\Omega(x-y)}{|x-y|^{n-1}}-\frac{\Omega(x+z-y)}{|x+z-y|^{n-1}}\right)\right. \\
& \left.\left.(b(x+z)-b(y)) f(y) d y\right|^{2} \frac{d t}{t^{3}}\right\}^{1 / 2} \\
\leq & C \int_{|x-y|>e^{\frac{1}{\varepsilon}}|z|}\left|\frac{\Omega(x-y)}{|x-y|^{n-1}}-\frac{\Omega(x+z-y)}{|x+z-y|^{n-1} \mid}\right| f(y) \mid\left\{\int_{\substack{|x-y| \leq t \\
|x+z-y| \leq t}} \frac{d t}{t^{3}}\right\}^{1 / 2} d y \\
\leq & C \int_{|x-y|>e^{\frac{1}{\varepsilon}}|z|}\left|\frac{\Omega(x-y)}{|x-y|^{n-1}}-\frac{\Omega(x+z-y)}{|x+z-y|^{n-1} \mid}\right| \frac{|f(y)|}{|x-y|} d y .
\end{aligned}
$$


Using Lemma 3.2, we get

$$
\begin{aligned}
& \left\{\frac{1}{r^{\lambda}} \int_{B(s, r)}\left(\int_{0}^{\infty}\left|J_{3}(x, t)\right|^{2} \frac{d t}{t^{3}}\right)^{p / 2} d x\right\}^{\frac{1}{p}} \\
& \leq C\left\{\frac{1}{r^{\lambda}} \int_{B(s, r)}\left(\int_{|x-y|>e^{\frac{1}{\varepsilon}}|z|}\left|\frac{\Omega(x-y)}{|x-y|^{n-1}}-\frac{\Omega(x+z-y)}{|x+z-y|^{n-1} \mid}\right| \frac{|f(y)|}{|x-y|} d y\right)^{p} d x\right\}^{1 / p} \\
& \leq C\|f\|_{p, \lambda} \int_{|y|>e^{\frac{1}{\varepsilon}}|z|}\left|\frac{\Omega(y)}{|y|^{n-1}}-\frac{\Omega(y+z)}{|y+z|^{n-1}}\right| \frac{1}{|y|} d y \\
& \leq C\|f\|_{p, \lambda} \sum_{k=0}^{\infty} \int_{2^{k} e^{\frac{1}{\varepsilon}}|z| \leq|y|<2^{k+1} e^{\frac{1}{\varepsilon}}|z|}\left|\frac{\Omega(y)}{|y|^{n-1}}-\frac{\Omega(y+z)}{|y+z|^{n-1}}\right| \frac{1}{|y|} d y \\
& \leq C\|f\|_{p, \lambda} \sum_{k=0}^{\infty}\left\{\frac{|z|}{2^{k} e^{\frac{1}{\varepsilon}}|z|}+\int \frac{|z|}{\frac{|z|}{2^{k} \frac{1}{\varepsilon}|z|}} \frac{\omega(\delta)}{\delta} d \delta\right\} \\
& \leq C\|f\|_{p, \lambda} \sum_{k=0}^{\infty}\left\{\frac{1}{2^{k} e^{\frac{1}{\varepsilon}}}+\frac{1}{1+k+1 / \varepsilon} \int_{\frac{1}{2^{k+1} e^{\frac{1}{\varepsilon}}}}^{\frac{1}{2^{k} e^{\frac{1}{\varepsilon}}}} \frac{\omega(\delta)}{\delta}(1+|\log \delta|) d \delta\right\} \\
& \leq C\left(e^{-\frac{1}{\varepsilon}}+\varepsilon\right)\|f\|_{p, \lambda} \\
& \leq C D \varepsilon \text {. }
\end{aligned}
$$

Thus

$$
\left\|\left\{\int_{0}^{\infty}\left|J_{3}(\cdot, t)\right|^{2} \frac{d t}{t^{3}}\right\}^{1 / 2}\right\|_{p, \lambda} \leq C D \varepsilon
$$

Now we give the estimate of $J_{4}$. Since $b \in C_{c}^{\infty}\left(\mathbb{R}^{n}\right)$, we have $|b(x)-b(x+z)| \leq$ $C|z|$. If set $\eta=e^{\frac{1}{\varepsilon}}|z|$ and

$$
\mu_{\Omega, \eta}(f)(x)=\left\{\int_{0}^{\infty}\left|\int_{\substack{|x-y|>\eta \\|x-y| \leq t}} \frac{\Omega(x-y)}{|x-y|^{n-1}} f(y) d y\right|^{2} \frac{d t}{t^{3}}\right\}^{1 / 2}
$$

then

$$
\left(\int_{0}^{\infty}\left|J_{4}(x, t)\right|^{2} \frac{d t}{t^{3}}\right)^{1 / 2} \leq C|z| \mu_{\Omega, \eta}(f)(x)
$$

We now claim that

$$
\left\|\mu_{\Omega, \eta}(f)\right\|_{p, \lambda} \leq C\|f\|_{p, \lambda}, \quad 1<p<\infty,
$$

where $C$ is independent of $\eta$ and $f$. In fact, if $B$ is a ball center at $x \in \mathbb{R}^{n}$ and of radius $\eta / 2$. Let $f_{1}(y)=f_{\chi_{2 B}}(y)$ and $f_{2}(y)=f(y)-f_{1}(y)$, then 


$$
\begin{aligned}
\mu_{\Omega, \eta}(f)(x) \leq & \frac{1}{|B|} \int_{B}\left|\mu_{\Omega}(f)(\xi)\right| d \xi+\frac{1}{|B|} \int_{B}\left|\mu_{\Omega}\left(f_{1}\right)(\xi)\right| d \xi \\
& +\frac{1}{|B|} \int_{B}\left|\mu_{\Omega}\left(f_{2}\right)(\xi)-\mu_{\Omega, \eta} f(x)\right| d \xi \\
\leq & \mathcal{M}\left(\mu_{\Omega}(f)\right)(x)+I(f)(x)+I I(f)(x) .
\end{aligned}
$$

By (2.6) and Lemma 3.3, we can get

$$
\left\|\mathcal{M}\left(\mu_{\Omega}(f)\right)\right\|_{p, \lambda} \leq C\left\|\mu_{\Omega}(f)\right\|_{p, \lambda} \leq C\|f\|_{p, \lambda} .
$$

Applying Theorem A, for any $1<u<\infty$

$$
I(f)(x) \leq \frac{C}{|B|^{1 / u}}\left\|\mu_{\Omega}\left(f_{1}\right)\right\|_{u} \leq \frac{C}{|B|^{1 / u}}\left\|f_{1}\right\|_{u} \leq C\left(\mathcal{M}\left(|f|^{u}\right)(x)\right)^{1 / u} .
$$

Taking $1<u<p$ and using Lemma 3.3 again, we have

$$
\|I(f)\|_{p, \lambda} \leq C\|f\|_{p, \lambda} .
$$

Regarding $I I(f)(x)$, let $\xi \in B$. By the Minkowski inequality, we have

$$
\begin{aligned}
& \left|\mu_{\Omega}\left(f_{2}\right)(\xi)-\mu_{\Omega, \eta}(f)(x)\right| \\
\leq & \left\{\int_{0}^{\infty}\left|\int_{\substack{|\xi-y| \geq t \\
|x-y| \leq t}} \frac{\Omega(x-y)}{|x-y|^{n-1}} f_{2}(y) d y\right|^{2} \frac{d t}{t^{3}}\right\}^{1 / 2} \\
& +\left\{\int_{0}^{\infty}\left|\int_{\substack{|\xi-y| \leq t \\
|x-y| \geq t}} \frac{\Omega(\xi-y)}{|\xi-y|^{n-1}} f_{2}(y) d y\right|^{2} \frac{d t}{t^{3}}\right\}^{1 / 2} \\
& +\left\{\int_{0}^{\infty}\left|\int_{\substack{|\xi-y| \leq t \\
|x-y| \leq t}}\left(\frac{\Omega(\xi-y)}{|\xi-y|^{n-1}}-\frac{\Omega(x-y)}{|x-y|^{n-1}}\right) f_{2}(y) d y\right|^{2} \frac{d t}{t^{3}}\right\}^{1 / 2} \\
:= & H_{1}(\xi, x)+H_{2}(\xi, x)+H_{3}(\xi, x) .
\end{aligned}
$$

Since $\xi \in B, y \in(2 B)^{c}$, similar to the estimate of (3.12), we may get

$$
\frac{1}{|B|} \int_{B} H_{1}(\xi, x) d \xi \leq C \eta^{1 / 2} \int_{(2 B)^{c}} \frac{|f(y)||\Omega(x-y)|}{|x-y|^{n+1 / 2}} d y .
$$

By the Minkowski inequality, for $s \in \mathbb{R}^{n}$ and $r>0$, we have

$$
\begin{aligned}
& \left\{\frac{1}{r^{\lambda}} \int_{B(s, r)}\left|\frac{1}{|B|} \int_{B} H_{1}(\xi, x) d \xi\right|^{p} d x\right\}^{\frac{1}{p}} \\
\leq & C \eta^{1 / 2}\left\{\frac{1}{r^{\lambda}} \int_{B(s, r)}\left(\int_{|y|>\eta} \frac{|f(x-y)||\Omega(y)|}{|y|^{n+1 / 2}} d y\right)^{p} d x\right\}^{\frac{1}{p}} \\
\leq & C \eta^{1 / 2}\|f\|_{p, \lambda} \int_{|y|>\eta} \frac{|\Omega(y)|}{|y|^{n+1 / 2}} d y \\
\leq & C\|f\|_{p, \lambda} .
\end{aligned}
$$


Thus

$$
\left\|\frac{1}{|B|} \int_{B} H_{1}(\xi, \cdot) d \xi\right\|_{p, \lambda} \leq C\|f\|_{p, \lambda} .
$$

For $H_{2}(\xi, x)$, we can get

$$
H_{2}(\xi, x) \leq C \eta^{1 / 2} \int_{(2 B)^{c}} \frac{|f(y)||\Omega(\xi-y)|}{|\xi-y|^{n+1 / 2}} d y
$$

Then

$$
\begin{aligned}
\frac{1}{|B|} \int_{B} H_{2}(\xi, x) d \xi & \leq C \eta^{1 / 2} \frac{1}{|B|} \int_{B} \int_{(2 B)^{c}} \frac{|f(y)||\Omega(\xi-y)|}{|\xi-y|^{n+1 / 2}} d y d \xi \\
& =C \eta^{1 / 2} \sum_{k=1}^{\infty} \frac{1}{|B|} \int_{B} \int_{2^{k+1} B \backslash 2^{k} B} \frac{|f(y)||\Omega(\xi-y)|}{|\xi-y|^{n+1 / 2}} d y d \xi \\
& \leq C \mathcal{M}(|f|)(x) .
\end{aligned}
$$

Thus, by Lemma 3.3, we have

$$
\left\|\frac{1}{|B|} \int_{B} H_{2}(\xi, \cdot) d \xi\right\|_{p, \lambda} \leq C\|f\|_{p, \lambda} .
$$

Since $\xi \in B$, we get

$$
\begin{aligned}
& \frac{1}{|B|} \int_{B} H_{3}(\xi, x) d \xi \\
& \leq \frac{1}{|B|} \int_{B} \int_{\mathbb{R}^{n}} \mid \frac{\Omega(\xi-y)}{|\xi-y|^{n-1}}-\frac{\Omega(x-y)}{|x-y|^{n-1} \mid \frac{\left|f_{2}(y)\right|}{|x-y|} d y d \xi} \\
& =\frac{1}{|B(0, \eta / 2)|} \int_{B(0, \eta / 2)} \int_{|y-x|>\eta \mid} \mid \frac{\Omega(x-\xi-y)}{|x-\xi-y|^{n-1}}-\frac{\Omega(x-y)}{|x-y|^{n-1} \mid} \frac{|f(y)|}{|x-y|} d y d \xi \\
& =\frac{1}{|B(0, \eta / 2)|} \int_{B(0, \eta / 2)} \int_{|y|>\eta}\left|\frac{\Omega(y-\xi)}{|y-\xi|^{n-1}}-\frac{\Omega(y)}{|y|^{n-1} \mid}\right| \frac{|f(x-y)|}{|y|} d y d \xi .
\end{aligned}
$$

By the Minkowski inequality and Lemma 3.2, for every $s \in \mathbb{R}^{n}$ and $r>0$, we get

$$
\begin{aligned}
& \left\{\frac{1}{r^{\lambda}} \int_{B(s, r)}\left|\frac{1}{|B|} \int_{B} H_{3}(\xi, x) d \xi\right|^{p} d x\right\}^{\frac{1}{p}} \\
\leq & C\|f\|_{p, \lambda} \frac{1}{|B(0, \eta / 2)|} \int_{B(0, \eta / 2)} \int_{|y|>\eta} \mid \frac{\Omega(y-\xi)}{|y-\xi|^{n-1}}-\frac{\Omega(y)}{|y|^{n-1} \mid} \frac{1}{|y|} d y d \xi \\
= & C\|f\|_{p, \lambda} \frac{1}{|B(0, \eta / 2)|} \int_{B(0, \eta / 2)} \sum_{k=1}^{\infty} \int_{2^{k-1} \eta<|y|<2^{k} \eta} \mid \frac{\Omega(y-\xi)}{|y-\xi|^{n-1}}-\frac{\Omega(y)}{|y|^{n-1} \mid} \frac{1}{|y|} d y d \xi
\end{aligned}
$$




$$
\begin{aligned}
& \leq C\|f\|_{p, \lambda} \frac{1}{|B(0, \eta / 2)|} \int_{B(0, \eta / 2)} \sum_{k=1}^{\infty}\left(\frac{|\xi|}{2^{k-1} \eta}+\int_{\frac{|\xi|}{2^{k_{\eta}}}}^{\frac{|\xi|}{2^{k-1} \eta}} \frac{\omega(\delta)}{\delta} d \delta\right) d \xi \\
& \leq C\|f\|_{p, \lambda} \frac{1}{|B(0, \eta / 2)|} \int_{B(0, \eta / 2)}\left(1+\int_{0}^{1} \frac{\omega(\delta)}{\delta} d \delta\right) d \xi \\
& \leq C\|f\|_{p, \lambda} .
\end{aligned}
$$

Thus

$$
\left\|\frac{1}{|B|} \int_{B} H_{3}(\xi, \cdot) d \xi\right\|_{p, \lambda} \leq C\|f\|_{p, \lambda} .
$$

Therefore

$$
\|I I(f)\|_{p, \lambda} \leq C\|f\|_{p, \lambda} .
$$

Summing up $\left\|M\left(\mu_{\Omega} f\right)\right\|_{p, \lambda},\|I(f)\|_{p, \lambda}$, and $\|I I(f)\|_{p, \lambda}$, by (3.17), we get (3.16).

Then

$$
\left\|\left\{\int_{0}^{\infty}\left|J_{4}(\cdot, t)\right|^{2} \frac{d t}{t^{3}}\right\}^{1 / 2}\right\|_{p, \lambda} \leq C|z|\|f\|_{p, \lambda} \leq C D|z| .
$$

About $J_{5}$, since $|b(x)-b(y)| \leq C|x-y|$, by the Minkowski inequality, we get

$$
\begin{aligned}
& \left\{\int_{0}^{\infty}\left|J_{5}(x, t)\right|^{2} \frac{d t}{t^{3}}\right\}^{1 / 2} \\
\leq & C \int_{|x-y| \leq e^{\frac{1}{\varepsilon}|z|}} \frac{|\Omega(x-y)|}{|x-y|^{n-1}}|b(x)-b(y)||f(y)|\left\{\int_{|x-y| \leq t} \frac{d t}{t^{3}}\right\}^{1 / 2} d y \\
\leq & C \int_{|x-y| \leq e^{\frac{1}{\varepsilon}|z|}} \frac{|f(y)||\Omega(x-y)|}{|x-y|^{n-1}} d y .
\end{aligned}
$$

Then by the Minkowski inequality and $\Omega \in L^{1}\left(S^{n-1}\right)$, for every $s \in \mathbb{R}^{n}$ and $r>0$, we get

$$
\left\{\frac{1}{r^{\lambda}} \int_{B(s, r)}\left(\int_{0}^{\infty}\left|J_{5}(x, t)\right|^{2} \frac{d t}{t^{3}}\right)^{p / 2} d x\right\}^{\frac{1}{p}} \leq C D e^{\frac{1}{\varepsilon}}|z| .
$$

Thus

$$
\left\|\left\{\int_{0}^{\infty}\left|J_{5}(\cdot, t)\right|^{2} \frac{d t}{t^{3}}\right\}^{1 / 2}\right\|_{p, \lambda} \leq C D e^{\frac{1}{\varepsilon}}|z| .
$$

Similarly, using the estimate $|b(x+z)-b(y)| \leq C|x+z-y|$, we have 


$$
\left\{\int_{0}^{\infty}\left|J_{6}(x, t)\right|^{2} \frac{d t}{t^{3}}\right\}^{1 / 2} \leq C \int_{|x-y| \leq e^{\frac{1}{\varepsilon}}|z|} \frac{|f(y)||\Omega(x+z-y)|}{|x+z-y|^{n-1}} d y .
$$

Then

$$
\left\|\left\{\int_{0}^{\infty}\left|J_{6}(\cdot, t)\right|^{2} \frac{d t}{t^{3}}\right\}^{1 / 2}\right\|_{p, \lambda} \leq C D\left(e^{\frac{1}{\varepsilon}}|z|+|z|\right) .
$$

Hence, for any $\varepsilon>0$, we may take $|z|$ to be small sufficiently, then by (3.10), (3.11), (3.13)-(3.15) and (3.18)-(3.20), we have

$$
\left\|\left[b, \mu_{\Omega}\right] f(\cdot+z)-\left[b, \mu_{\Omega}\right] f(\cdot)\right\|_{p, \lambda} \leq C \varepsilon .
$$

Therefore, we show that (3.2) holds for the commutator $\left[b, \mu_{\Omega}\right]$ in $G$ uniformly and complete the proof of Theorem 2 .

\section{REFERENCES}

1. A. Benedeck, A. P. Calderon and R. Panzone, Convolution operators on Banach space valued functions, Proc. Nat. Acad. Sci. USA, 48 (1962), 356-365.

2. M. S. Berger, Nonlinearity and Functional Ananysis, New York, Academic Press, 1977, pp. 64-107.

3. Y. Chen and Y. Ding, Compactnesss of commutators for Littlewood-Paley operators, Kodai Math. J., 32 (2009), 256-323.

4. Y. Chen, Y. Ding and X. Wang, Compactness characterization of commutators for singular integrals on Morrey spaces, Preprint.

5. R. Coifman, R. Rochberg and G. Weiss, Factorization theorems for Hardy spaces in several variables, Ann. Math., 103 (1976), 611-635.

6. L. Colzani, Hardy spaces on sphaere, Ph.D. Thesis, Washington University, St. Louis, MO, 1982.

7. Y. Ding, A note on end properties of Marcinkiewicz integral, J. Korean Math. Soc., 42 (2005), 1087-1100.

8. Y. Ding, D. Fan and Y. Pan., $L^{p}$ boundedness of Marcinkiewicz integrals with Hardy space function kernels, Acta Math. Sin. Ser. B (English), 16 (2000), 593-600.

9. Y. Ding and S. Lu, Homogeneous fractional integrals on Hardy spaces, Tôhoku Math. J., 52 (2000), 153-162.

10. Y. Ding, S. Lu and K. Yabuta, On commutator of Marcinkiewicz integrals with rough kernel, J. Math. Anal. Appl., 275 (2002), 60-68.

11. S. Janson, Mean oscillation and commutators of singular integral operators, Ark. Mat., 16 (1978), 263-270. 
12. T. Mizuhara, Boundedness of some classical operators on generalized Morrey spaces, Harmonic Analysis, ICM-90 Satellite Conf. Proc., S. Igari (ed.), Springer-Verlag, Tokyo, 1991, pp. 183-189.

13. C. Morrey, Sur quelques intégrales du type de Dini, Ann. Soc. Poloa. Math., 17 (1938), 42-50.

14. E. M. Stein, On the function of Littlewood-paley, Lusin and Marcinkiewicz, Trans. Amer. Math. Soc., 88 (1958), 430-466.

15. E. M. Stein, Singular Integrals and Differentiability Properties of Functions, Princeton Univ. Press. Princeton, N.J. 1970.

16. E. M. Stein, Harmonic Analysis: Real Variable Methods, Orthogonality, and Oscillatory Integrals, Princeton Univ. Press, Princeton, 1993.

17. E. M. Stein and G. Weiss, Introdution to Fourier analysis on Euclidean spaces, Princeton University Press, Princeton, 1971.

18. A. Uchiyama, On the compactness of operators of Hankel type, Tôhoku Math., 30 (1976), 163-171.

Yanping Chen

Department of Mathematics and Mechanics

Applied Science School

University of Science and Technology Beijing

Beijing 100083

P. R. China

E-mail: yanpingch@126.com

Yong Ding

School of Mathematical Sciences

Laboratory of Mathematics and Complex Systems (BNU)

Minstry of Education of China

Beijing Normal University

Beijing 100875

P. R. China

E-mail: dingy@bnu.edu.cn

Xinxia Wang

The College of Mathematics and System Science

Xinjiang University

Urumqi, Xinjiang, 830046

P. R. China

E-mail: wxxa@xju.edu.cn 